\title{
Directional solidification casting technology of heavy-duty gas turbine blade with liquid metal cooling (LMC) process
}

\author{
*Xiao-fu Liu, Yan-chun Lou, Bo Yu, Gui-qiao Su, Chang-chun Li, Xin-li Guo, Biao Li, and Guo-yan Shui \\ State Key Laboratory of Light Alloy Casting Technology for High-end Equipment, Shenyang Research Institute of Foundry Co., Ltd., Shenyang \\ 110022, China
}

\begin{abstract}
In this work, some important factors such as ceramic shell strength, heat preservation temperature, standing time and withdrawal rate, which influence the formability of directionally solidified large-size blades of heavy-duty gas turbine with the liquid metal cooling (LMC) process, were studied through the method of microstructure analysis combining. The results show that the ceramic shell with medium strength (the high temperature flexural strength is $8 \mathrm{MPa}$, the flexural strength after thermal shock resistance is $12 \mathrm{MPa}$ and the residual flexural strength is $20 \mathrm{MPa}$ ) can prevent the rupture and runout of the blade. The appropriate temperature $\left(1,520{ }^{\circ} \mathrm{C}\right.$ for upper region and $1,500{ }^{\circ} \mathrm{C}$ for lower region) of the heating furnace can eliminate the wide-angle grain boundary, the deviation of grain and the run-out caused by the shell crack. The holding time after pouring (3-5 min) can promote the growth of competitive grains and avoid a great deviation of columnar grains along the crystal orientation $<001>$, resulting in a straight and uniform grain structure. In addition, to avoid the formation of wrinkles and to ensure a smooth blade surface, the withdrawal rate should be no greater than the growth rate of grain. It is also found that the dendritic space of the blade decreases with the rise of solidification rate, and increases with the enlarging distance between the solidification position and the chill plate.
\end{abstract}

Key words: liquid metal cooling (LMC); heavy-duty gas turbine; large-size blade; directional solidification; microstructure

CLC numbers: TG132.3 3 Document code: A Article ID: 1672-6421(2019)01-023-08

Gequipment for the high-efficiency energy
transformation and clean utilization systems. Turbine
blades, as the core components of the gas turbines, are
more and more difficult to manufacture due to the higher
requirement for the increasing power generation capacity
and power efficiency. The superalloy turbine blades
developed from the forged blades and equiaxed grain
casting blades to the directionally solidified columnar
grain blades and also single crystal blades. During the
development, the heat resistance of the superalloy blades
increased by $\sim 400{ }^{\circ} \mathrm{C}$ (i.e. $\sim 6.7{ }^{\circ} \mathrm{C}$ per year on average)
and the operation temperature increased from $700{ }^{\circ} \mathrm{C}$
for forged blades to $1,050-1,100{ }^{\circ} \mathrm{C}$ for single crystal

Male, born in 1979, Ph.D, Senior Engineer. His research is mainly focused on investment casting of superalloys.

E-mail: liuxf-01@163.com blades ${ }^{[1]}$. In addition, combining the gas cooling and thermal barrier coating, the heat resistance of superalloy blades could be as high as $1,500-1,700{ }^{\circ} \mathrm{C}{ }^{[2]}$, which greatly improved the thrust-weight ratio of aero-engine and the power generation efficiency of the industrial gas turbines.

At present, there are two methods to manufacture the directionally solidified columnar grain blade and the single crystal blade, i.e. high rate solidification (HRS) (Fig. 1a) and liquid metal cooling (LMC) (Fig. 1b). LMC originated from $1974{ }^{[3]}$ and it is still a promising directional solidification technology. In this process, the ceramic shell and the melt are taken out from the mold heating furnace and quenched in a liquid metal coolant with a cooling rate that two times faster than that of the HRS ${ }^{[4]}$, resulting in a higher thermal gradient and a solidification rate. This helps overcome the weakness of HRS, such as low thermal gradient and lack of stability. LMC method has a high thermal gradient of $60-80{ }^{\circ} \mathrm{C} \cdot \mathrm{cm}^{-1}$ in general, which is up to $200{ }^{\circ} \mathrm{C} \cdot \mathrm{cm}^{-1}$ in some particular cases ${ }^{[5]}$. Thus the large-size blades of 

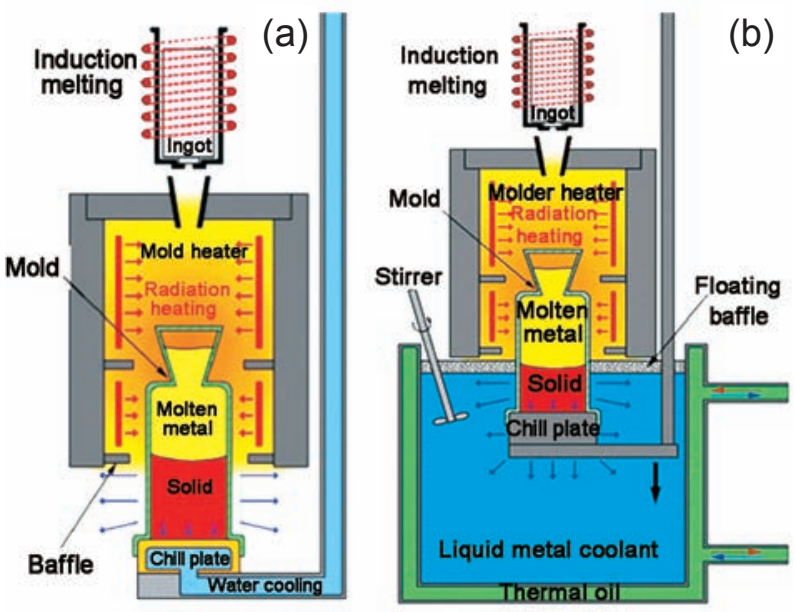

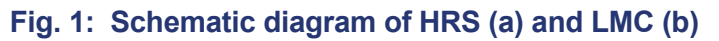

heavy-duty gas turbine made by LMC contain spurious grains, lower porosity, and is in a finer and more homogeneous structure compared with that made by HRS process ${ }^{[6-7]}$.

The dramatic temperature drop is a great challenge to the thermal shock resistance of the ceramic shell. Besides, a largesize blade is more likely to be deformed or crack because it undergoes greater impact from the alloy melt and higher static pressure after pouring. Therefore, the influence of different shell strengths on the formability of the large-size hollow turbine blade should be studied. In the directional solidification process, the refined superalloy melt is poured slowly into the ceramic shell, which is heated by the mold heating furnace and positioned onto the chill plate. Then, the melt is withdrawn downward after a period of time for standing, for which the duration influences the formation of directional solidification structure. There are two advantages of holding: First, it allows the superalloy melt to achieve a uniform body temperature and prevent segregation. Second, it induces a competition among the growing crystal grains, which helps the formation of columnar grain with a good quality. In previous research, the holding time was usually set to be $3-5 \mathrm{~min}$, but the reasoning, and how the holding time influences the formation of grain structure in casting were not discussed. The withdrawal rate and crystal grain growth rate are required to be as consistent as possible and well match the formation of a blade with ideal structure.

In this work, the directional solidification technique for making the large-size blade for a heavy-duty gas turbine (Grade II ) under the LMC conditions was studied, including the effects of ceramic shell strength and some important process factors, such as the heat preservation temperature, holding time and withdrawal rate on the directional solidification formation of gas turbine blade.

\section{Experimental method}

The large-size blades of heavy-duty gas turbine (Grade II) were made of DZ466 superalloy by using the ZGD-25BYF LMC (Sn coolant) furnace. As shown in Fig. 2, the total length of the blade is about $360 \mathrm{~mm}$ and the width of the chord is about $160 \mathrm{~mm}$.

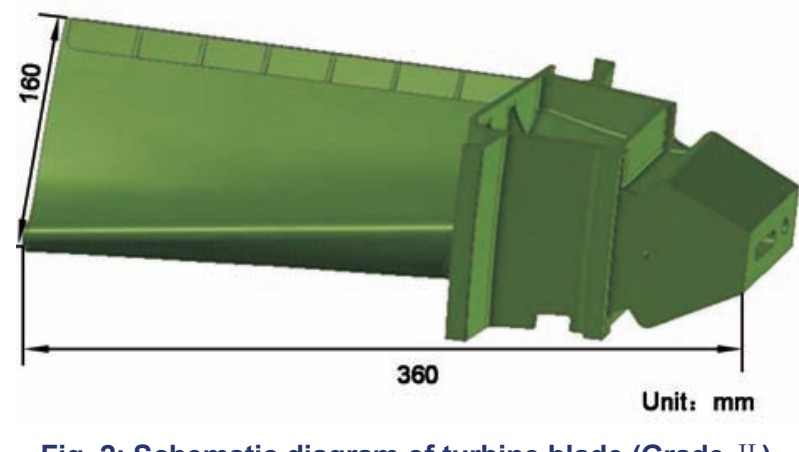

To investigate the influence of different shell strengths on the formability of the large-size hollow turbine blade, the high, low, and medium strength ceramic shells were applied in the directional solidification process. The strengths of the ceramic shell were measured by using WDW-1 type flexural strength instrument and the results are shown in Table 1.

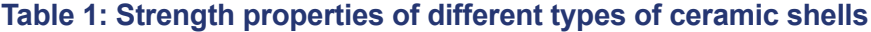

\begin{tabular}{|cccc} 
& High temperature flexural & Flexural strength after & Residual flexural strength \\
\hline High strength ceramic shell & 10 & 15 & 30 \\
Low strength ceramic shell & 6 & 8 & 14 \\
Medium strength ceramic shell & 8 & 12 & 20
\end{tabular}

The heat preservation temperature of the mold heating furnace plays an important role in controlling the thermal gradient of the solid-liquid interface during the directional solidification process. Three conditions were tested to investigate how the heat preservation temperature influences the formability of the blade (Table 2)

The holding time was set to be 3, 5 and $8 \mathrm{~min}$, to investigate how the holding time influences the formation of grain structure in casting. The pouring temperature was $1,520^{\circ} \mathrm{C}$. Then, with

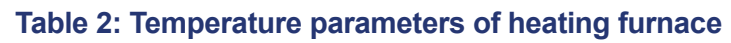

\begin{tabular}{|c|c|c|}
\hline 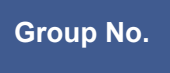 & 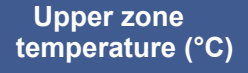 & 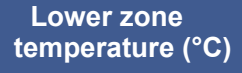 \\
\hline 1 & 1,460 & 1,480 \\
\hline 2 & 1,500 & 1,520 \\
\hline 3 & 1,540 & 1,560 \\
\hline
\end{tabular}


the shell mold was withdrawn at different speeds of 3, 5, 7 and $10 \mathrm{~mm} \cdot \mathrm{min}^{-1}$ to investigate their effects on the formability of the blade casting. Finally, the shell molds were submerged into a Sn (tin) coolant $\left(260-280^{\circ} \mathrm{C}\right)$ to realize the directional solidification of the blade.

To observe the microstructures and check the wall thicknesses of the blade, samples from five different locations of the directionally solidified superalloy blade were taken. As shown in Fig. 3, the solidification order of the blade was $\# 5 \rightarrow \# 4 \rightarrow \# 3 \rightarrow \# 2 \rightarrow \# 1$. Sample \#1 is a last-solidified rabbet part of the blade with a thickness of $\sim 10 \mathrm{~mm}$ (including the thickness of ceramic core). Sample \#2 is a boss part of the blade with a thickness of $\sim 7 \mathrm{~mm}$. Sample \#3 is a reinforced rib in the blade with a thickness of $\sim 7 \mathrm{~mm}$. Sample \#4 is a thin-walled part of the blade with a thickness of $\sim 2 \mathrm{~mm}$. Sample \#5 is a firstsolidified crystal induction section of the blade with a thickness of $\sim 10 \mathrm{~mm}$

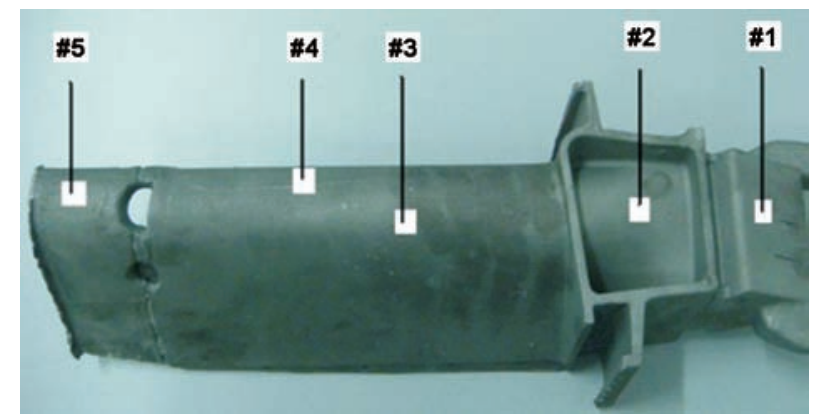

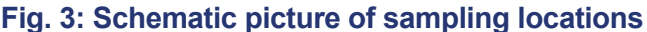

The columnar grain structure of the initial grains was observed at $0,3,5$ and 8 min after the pouring. A mixture of $\mathrm{H}_{2} \mathrm{O}_{2}$ and $\mathrm{HCl}$ with a volume ratio of 1:1 was applied for etching the directional macrostructures of the blades, which was observed using an optical microscope (OM). Both transversal and longitudinal structures were observed. In addition, Hitachi scanning electron microscope (SEM) (Model $\mathrm{S}-3400 \mathrm{~N}$ ) with $20 \mathrm{kV}$ sweep voltage, $3 \mathrm{~nm}$ resolution and 500-5,000 times magnification, was applied to observe the fracture morphologies of the blades.
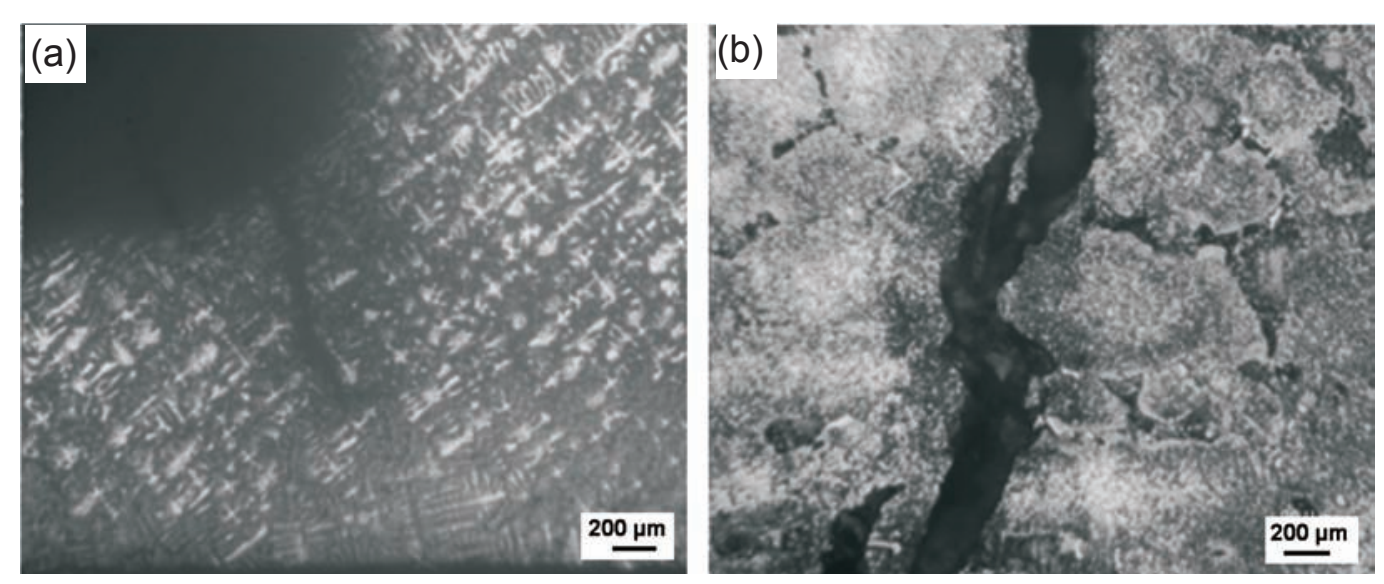

Fig. 5: Cracking morphology of directional solidification blade by OM

\section{Results and discussion}

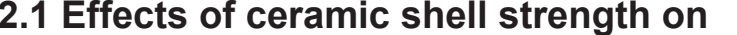

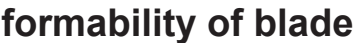

The high strength ceramic shell was used and its strength properties are shown in Table 1. The experiment results show that the hollow turbine blades were broken (Fig. 4), and this is because the contraction stress, introduced in the directional solidification of the blade, doesn't have enough time to be released due to the high strength of ceramic shell. When the stress is greater than the maximum strength of the alloy, the directional hollow blade will crack during the solidification, resulting in incomplete formation of the blade.

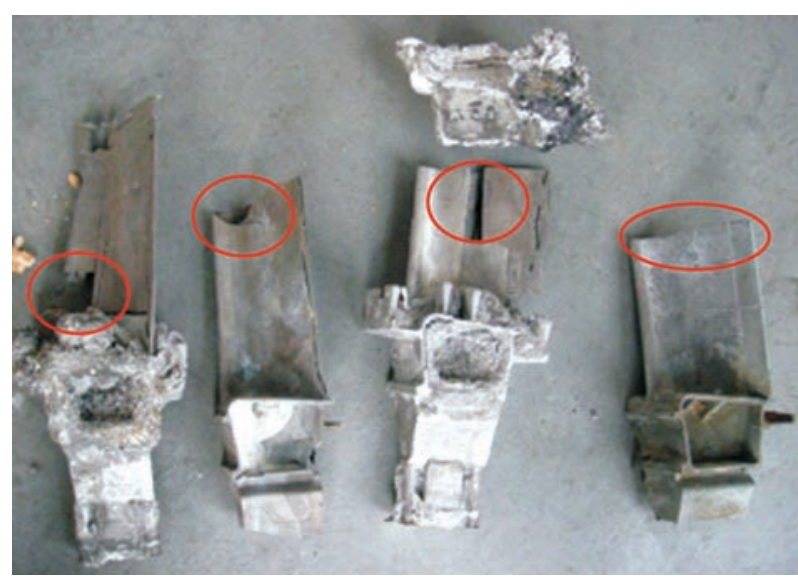

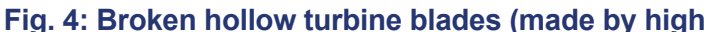

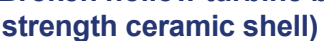

The cracking was characterized to be transgranular fracture observed by OM, as shown in Fig. 5, and the intergranular fracture was not found. The cracking morphology was further studied through SEM, in which a herringbone pattern can be observed in both transversal and longitudinal fractures, indicating a brittle property (Fig. 6). The pattern suggests that the cracks occured during a shape contraction after the solidification. It is actually a cold crack caused by the poor collapsibility and the high residual strength of the ceramic shell. 

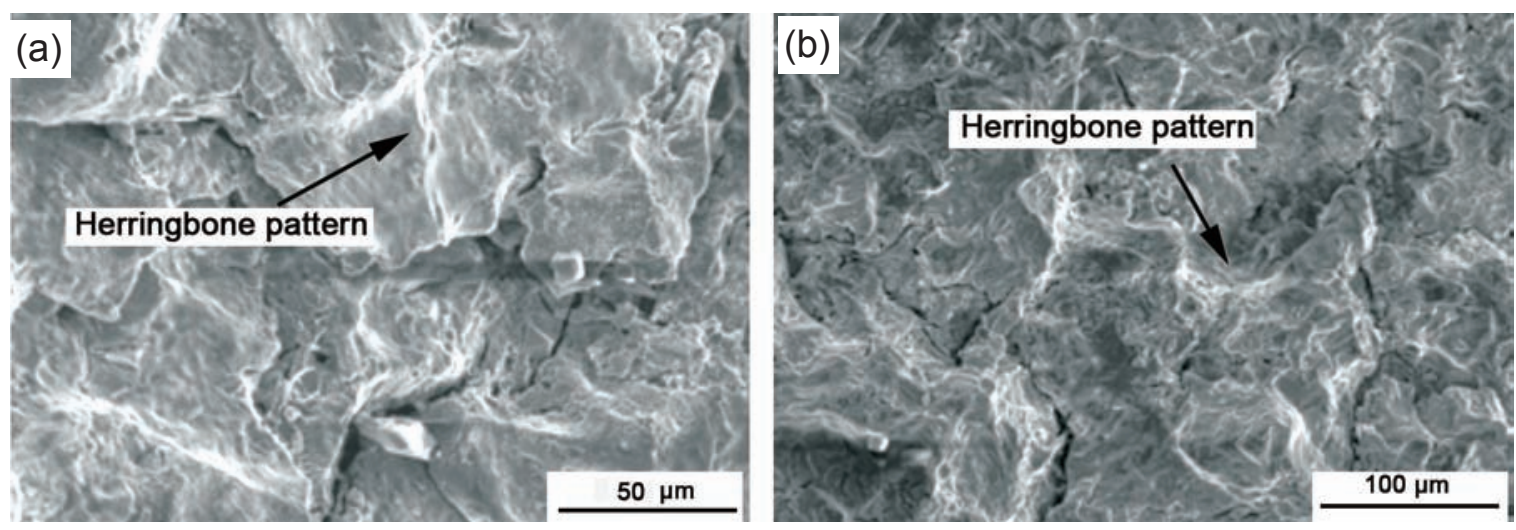

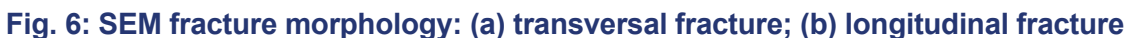

The low strength ceramic shell was also applied in the directional solidification process, and the runout occurred, as shown in Fig. 7. This is because the strength of the ceramic shell is too low to withstand the dynamic and static pressure of the liquid superalloys. As a result, the alloy melt is easily leaks from the exhaust side, causing a runout.
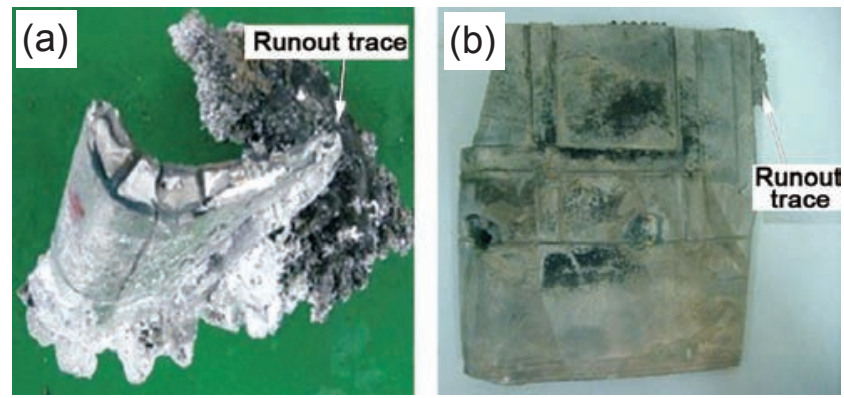

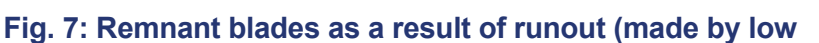

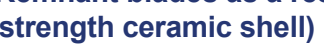

Then, the medium strength ceramic shell was tried, whose strength properties are shown in Table 1. This ceramic shell showed a good deformability, which reduces the contraction resistance force and the crack tendency of the blade. Figure 8 shows the complete hollow turbine blade made by the medium strength ceramic shell. It can be seen that the ceramic shell with this level of strength is suitable for the formation of directionally solidified turbine blade under LMC conditions.

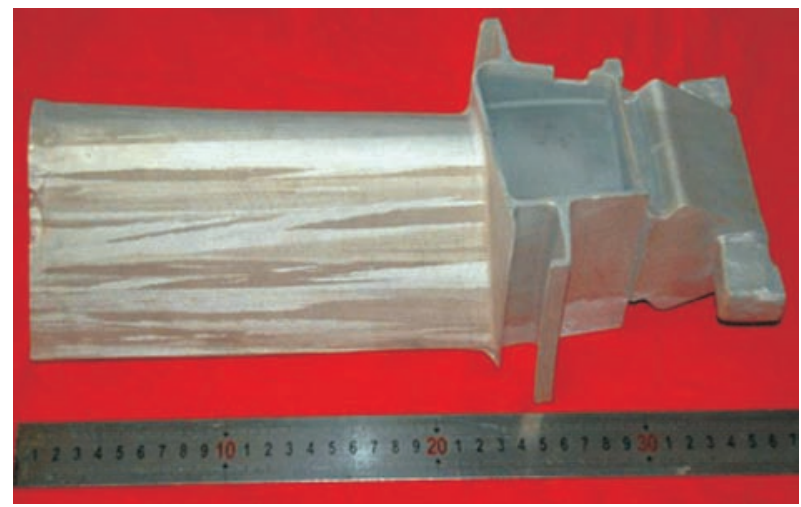

Fig. 8: Complete formation of directionally solidified

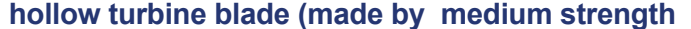

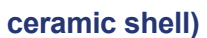

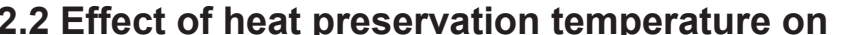

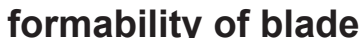

The medium strength ceramic shell was used to study the effect of heat preservation temperature on the formability of blade. The defective blade [Fig. 9(a)] was obtained under the heat preservation temperature of Group 1 in Table 2. The higher angle of grain boundary and deviation of crystal grain in the blade can be seen in the defective blade. This is because of a lower degree of superheating in the superalloy melt and lower thermal gradient at the interface of solid and liquid, both of which were produced by the lower heat preservation temperature. The higher heat preservation temperature (Group 3 in Table 2) makes the ceramic shell soften and crack, resulting in some leakage of melt [Fig. 9(b)]. A complete blade can be achieved in the condition with medium heat preservation temperature (Group 2 in Table 2) as shown in Fig. 9(c). This proves that an appropriate heat preservation temperature (the upper zone temperature of the mold heating furnace was $1,500{ }^{\circ} \mathrm{C}$, and the lower zone was $1,520^{\circ} \mathrm{C}$ ) can prepare a sound blade.
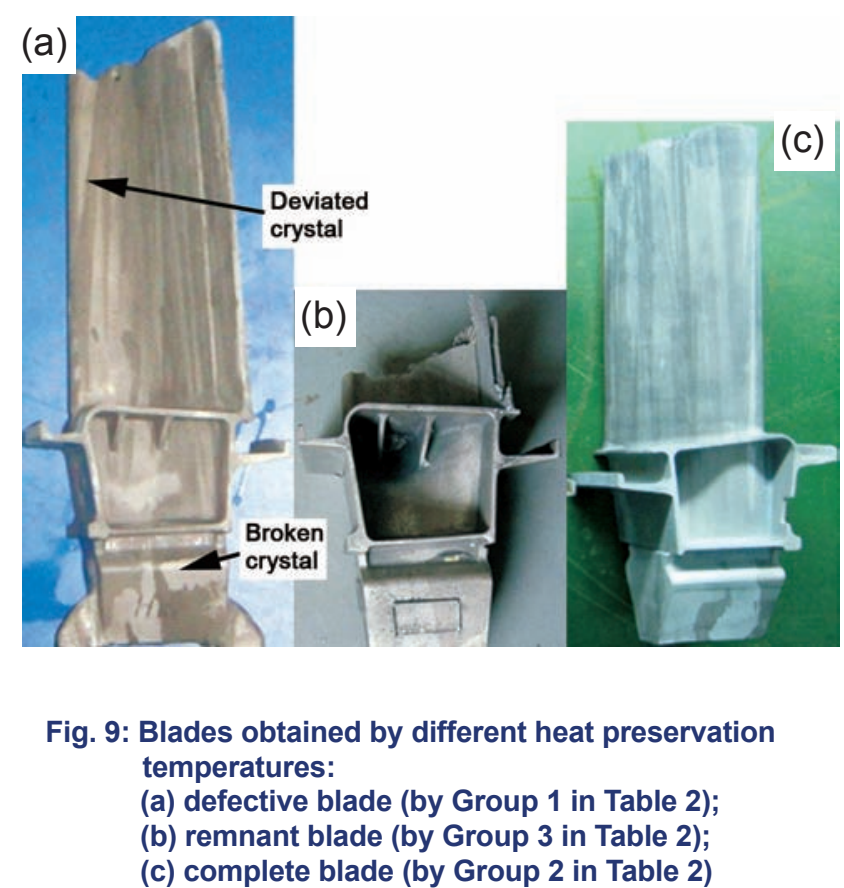


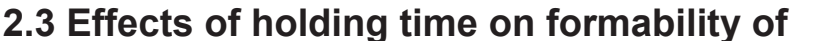
पाII $\square \square$

The medium strength ceramic shell and holding temperature of Group 2 in Table 2 were used. When the holding time is 0 min [Fig. 10(a)], the initial equiaxed crystal grain grows in the direction of spontaneous nucleation without screening through the competition. Therefore, the growing direction of crystal are not parallel with that of the heat flow, resulting in a narrow shape columnar grain and a great deviation of orientation from $<001>$ in the blade.

When the holding times are $3 \mathrm{~min}$ and $5 \mathrm{~min}$ (Fig. 10b and c), the initial equiaxed crystal grains undergo a competitive screening during the growth. During this process, the crystal grains with the orientation deviated from the direction of heat flow are eliminated. As a result, the remaining grains are in a good quality with fine and straight columnar shape. These grains grow in a steady state, which can be expressed as ${ }^{[8]}$ :

$$
K_{\mathrm{s}} G_{\mathrm{s}}=K_{\mathrm{L}} G_{\mathrm{L}}+\Delta H_{\mathrm{F}} \rho V+\Delta E V
$$

where, $K$ is the thermal conductivity $\left[\mathrm{W} \cdot(\mathrm{m} \cdot \mathrm{K})^{-1}\right]$, the subscript $\mathrm{S}$ is the solid, the subscript $\mathrm{L}$ is the liquid, $G_{\mathrm{L}}$ is the thermal gradient at solidification front $\left(\mathrm{K} \cdot \mathrm{m}^{-1}\right), G_{\mathrm{S}}$ is the gradient in solid $\left(\mathrm{K} \cdot \mathrm{m}^{-1}\right), \Delta H_{\mathrm{F}}$ is the latent heat $\left(\mathrm{kJ} \cdot \mathrm{kg}^{-1}\right), \Delta E$ is the energy of unit volume melt absorbed from exterior $\left(\mathrm{kJ}^{-1} \mathrm{~mol}^{-1}\right), \rho$ is the density $\left(\mathrm{kg} \cdot \mathrm{m}^{-3}\right), V$ is the solidification rate $\left(\mathrm{m} \cdot \mathrm{s}^{-1}\right)$.

$G_{\mathrm{L}}$ and $V$ are correlated and difficult to calculate accurately, especially when the radial heat flow is involved. In the equation (1), $K, \rho, \Delta H$ and $\Delta E$ are approximately constant values once the components of alloy are determined. In the initial stage of solidification, $G_{\mathrm{L}}$ can be up to $100{ }^{\circ} \mathrm{C} \cdot \mathrm{cm}^{-1}$, while $G_{\mathrm{s}}$ is quite low, which brings an equilibrium state for the solidification. The low growth rate allows grain columns to have more time to achieve a good quality and a desired morphology.
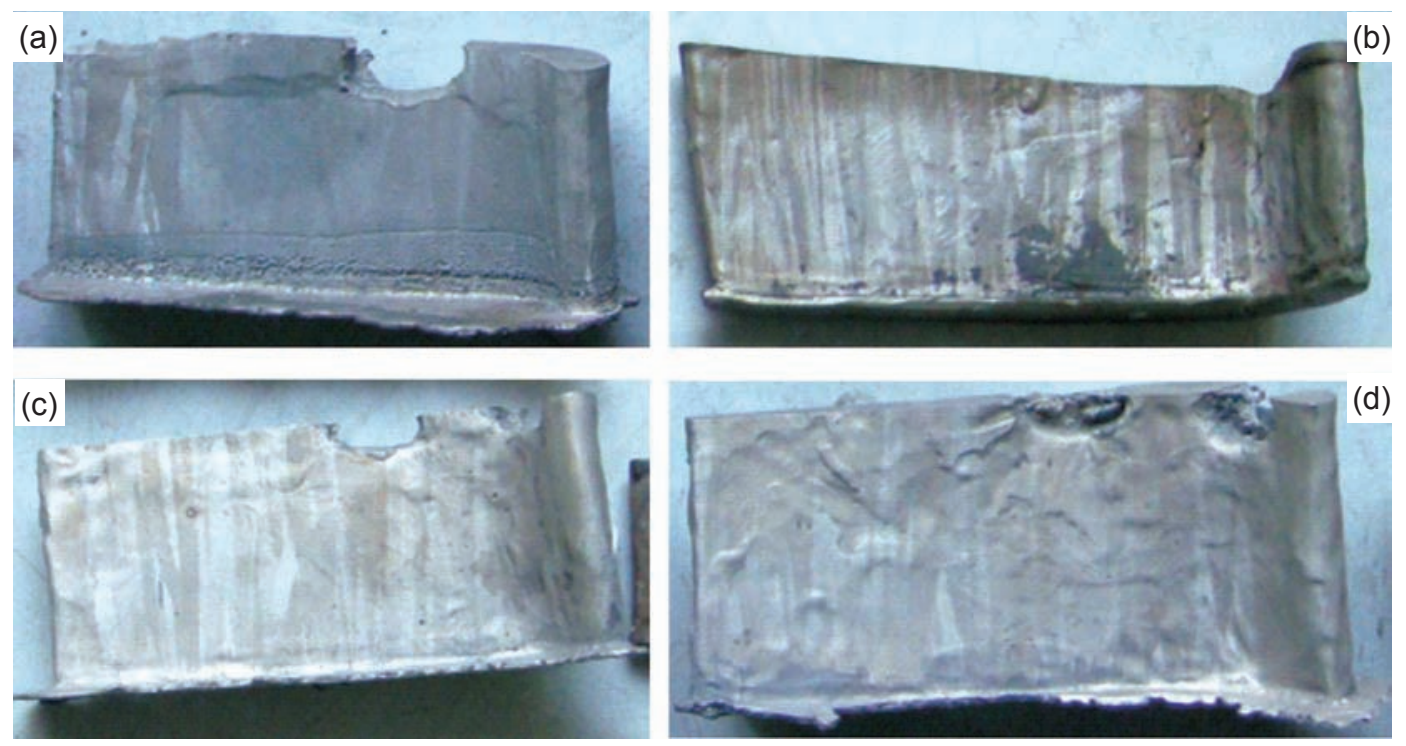

Fig. 10: Original structures of blades with various holding times: (a) $0 \mathrm{~min}$; (b) $3 \mathrm{~min}$; (c) $5 \mathrm{~min}$; (d) $8 \mathrm{~min}$

When the holding time was 8 min [Fig. 10(d)], due to the long incubation time, transversely grown grains occur and cause the repeated melting of the top ends of the dendrites. The broken dendrites without being melted become a new nuclei that later on grow in their original directions, resulting in broader shape grain columns with a serious crystal deviation. Therefore, holding time should be $3 \mathrm{~min}$ or $5 \mathrm{~min}$.

Figure 11 shows the relationship between the holding time and the original crystal grain numbers. It can be seen from this figure that the largest number, which could be over 30 , of initial crystal grains occurs when the holding time is $3 \mathrm{~min}$. The smallest number is below 15 , which occurs when there is no holding time. Five minutes and 8 min holding times result in a medium number of initial crystal grains.

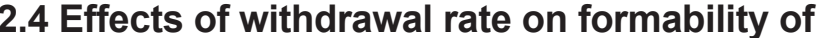
पाएव

Figure 12(a) and (b) show a large-size hollow blade made by the withdrawal rate of 3 and $5 \mathrm{~mm} \cdot \mathrm{min}^{-1}$, respectively. And the blades with a smooth surface quality can be obtained under

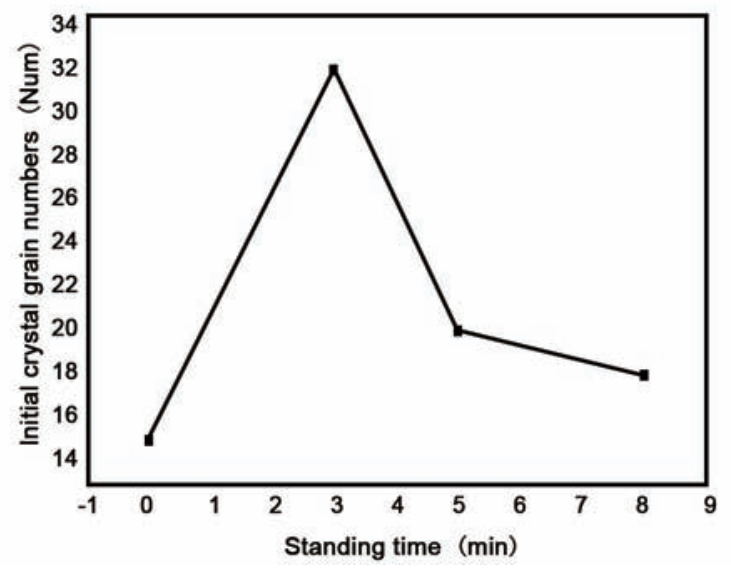

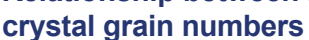



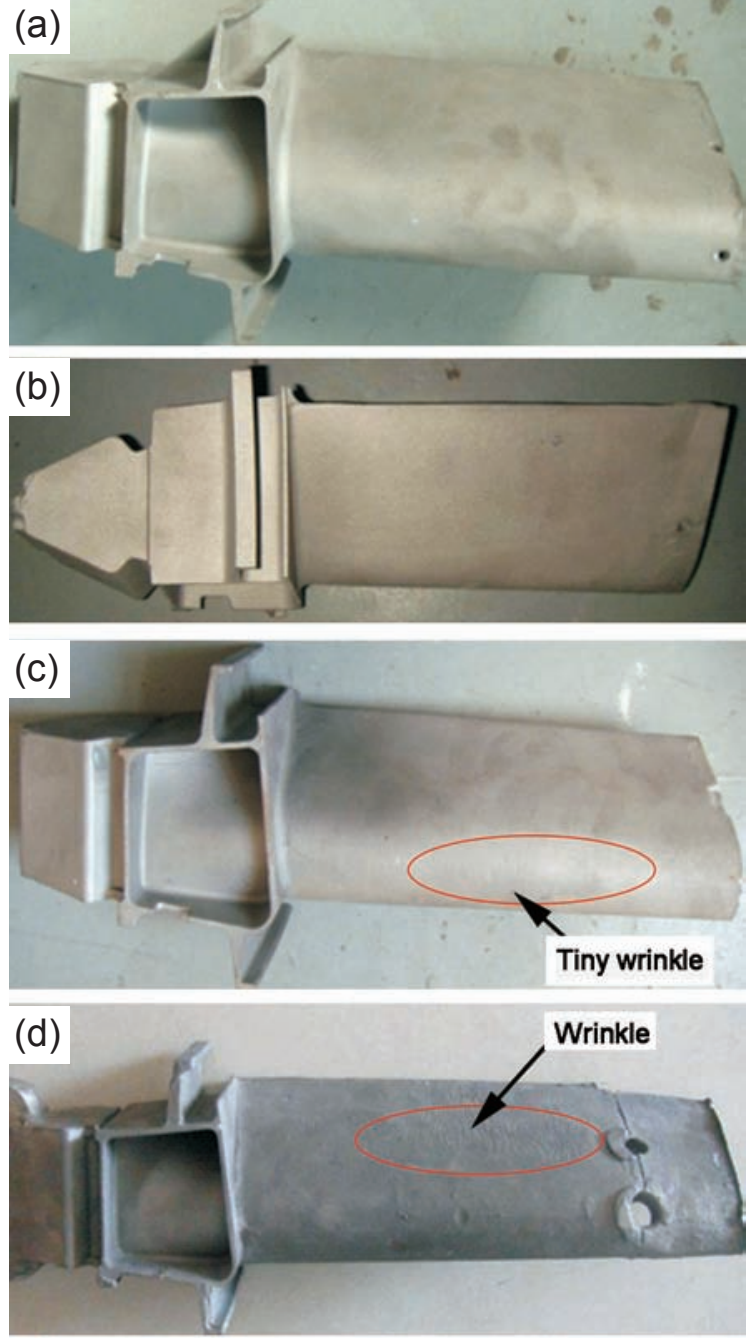

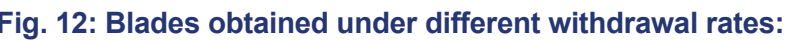

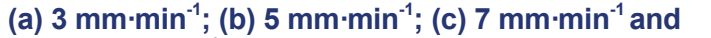

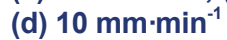

those two withdrawal rates. However, when the withdrawal rates were as high as 7 and $10 \mathrm{~mm} \cdot \mathrm{min}^{-1}$, the surface quality reduces. Meanwhile, wrinkles with different depths occurred (Fig. 12c and d) because of the excessive withdrawal rate although the complete formation of blade was obtained .

After the pouring of the superalloy melt, gaps existed between the solidified metal and ceramic shell due to the shrinkage of the metal that occurred during the withdrawal process. Under the effect of pressure, tin melt entered the gap along the interface between the bottom surface of ceramic shell and the chill plate. When the withdrawal rate is not greater than the growth rate of crystal grain, the solidification interface is at the same level with or higher than the liquid tin. Consequently, the liquid tin remains on the surface of solidified superalloy blade without any impact on the surface property and can be removed by the surface sand blasting treatment after the cooling. When the withdrawal rate is greater than the crystal grain growth rate, the solidification interface is lower than that of the liquid tin. The pressure difference between the liquid tin that stays on the surface of ceramic shell and that exists at the solidification interface of the alloy inside the ceramic shell, can be expressed as $\rho g \Delta H$ (Fig. 13). The liquid tin between the ceramic shell and the solidified metal goes upward and hits the non-solidified superalloy melt under the pressure difference. Simultaneously, the ceramic shell keeps descending, which ensures a constant scouring of the liquid tin against the nonsolidified superalloy melt. During this process, the heat of the melt is also transferred to the ceramic shell via the liquid tin. As a result, crimps are formed during the solidification where the superalloy melt contacts with the ceramic shell and the liquid tin.

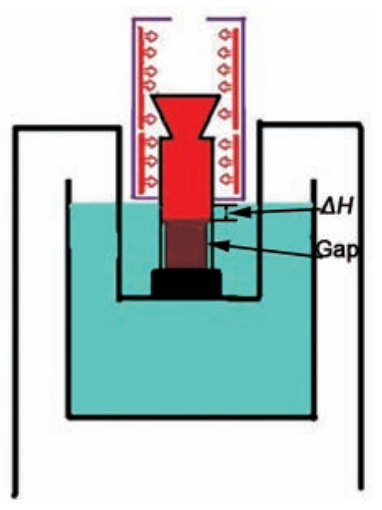

Fig. 13: Relationship among ceramic shell, solidified metal and solidification interface

\subsection{Microstructure of directionally solidified blade}

Figure 14 shows the transversal and longitudinal structures of the samples from different locations of the blade. Transversal structures of all samples present the pattern like "+" (Fig. $14 \mathrm{a}, \mathrm{c}, \mathrm{e}, \mathrm{g}$ and $\mathrm{i}$ ), but with the great difference in the dendrite space (shown in Fig. 15). The dendrite spaces of sample \#1 are in the range of 250-320 $\mu \mathrm{m}$ but relatively homogeneous (Fig. 14a). For sample \#2, the dendrite spaces are about 200-280 $\mu \mathrm{m}$, showing great variance (Fig. 14c). Sample \#3 has dendrite spaces about 180-260 $\mu \mathrm{m}$ and transversal structures with a pattern like "dragonfly" (Fig. 14e). The well developed primary dendrites demonstrate the existence of transverse thermal gradient. Due to the great curvature and large specific surface area of the blade back, some of the heat firstly transferred to liquid tin via the ceramic shell along the transverse direction. The dendrite spaces of sample \#4 are about 150-240 $\mu \mathrm{m}$ and quite homogeneous (Fig. 14g). The dendritic structure of sample \#5 is very fine (Fig. 14i), and the spaces are $80-120 \mu \mathrm{m}$. The sample \#5 is at a position where crystal grains begin to nucleate. In this case, after the superalloy melt was poured into the ceramic shell, the melt that contacts with the graphite chill plate was solidified instantly because of the large thermal gradient, and resulting in a fine dendritic structure. During the directional withdrawal process, the cooling effect of the chilling plate on the melt weakened gradually and finally disappeared. The thermal gradient at the front edge of the solidification interface also decreased gradually, leading to a coarser dendritic structure.

The longitudinal structures of samples \#1-4 (Fig. 14b, d, f and h) all present a pattern like "sugar gourd string" with clear 

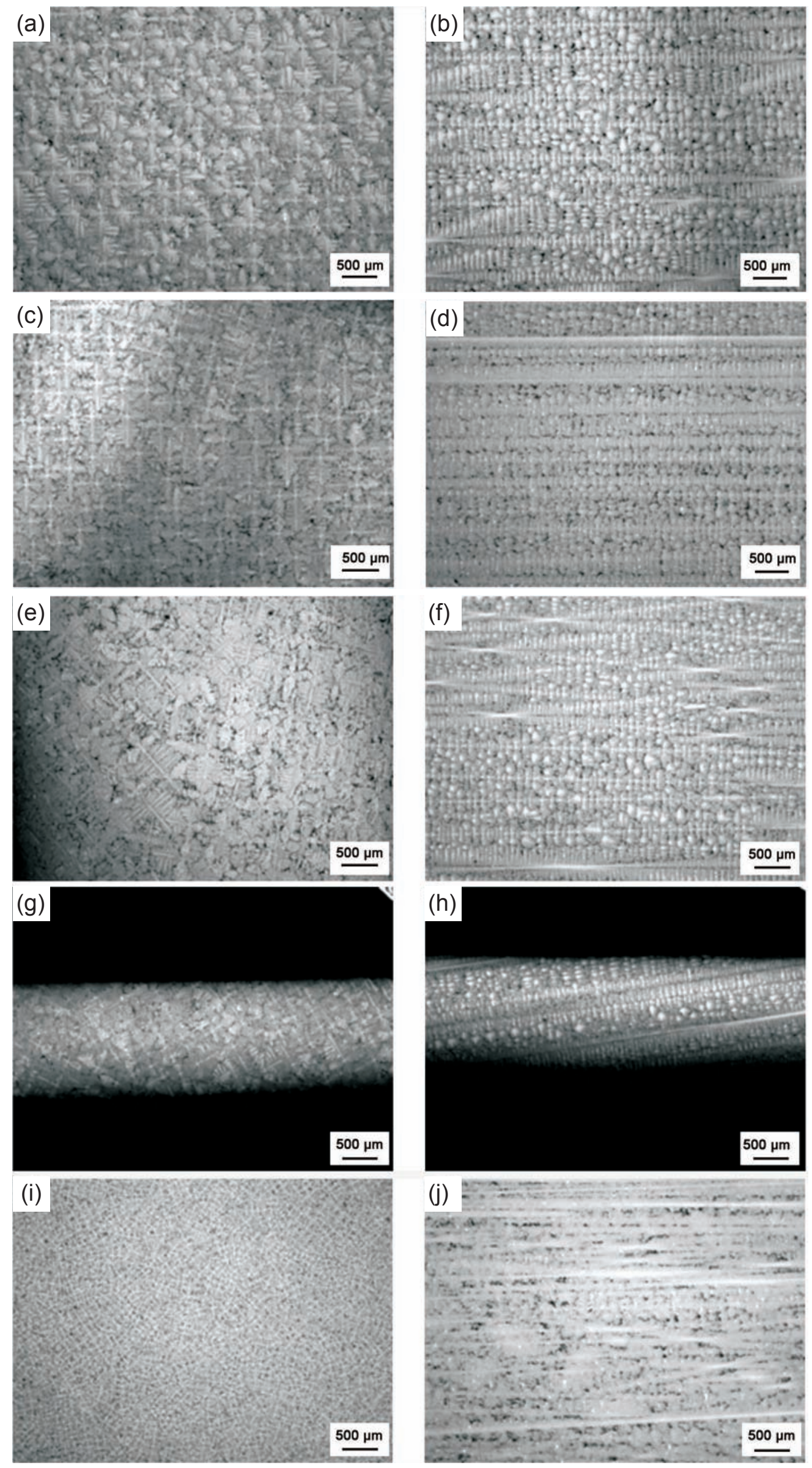

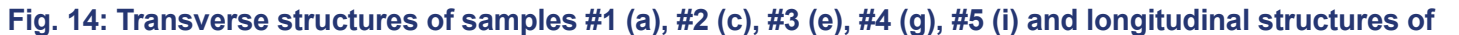

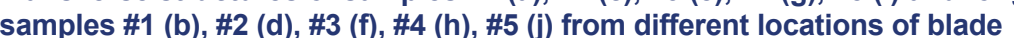


structures of the primary dendrite. The secondary dendrites of sample \#1 (Fig. 14b) were well developed because that during the later period of the withdrawal process, the blade became thicker and the thermal gradient decreased, and thus the heat transfer rate along the longitudinal direction was not sufficient. Meanwhile, the transverse thermal gradient occured, resulting in a certain angle between the direction of heat flow and that of the crystal grain growth. The secondary dendrite grew in the opposite direction to the heat flow, while, the primary dendrites still grew in the original direction due to the inertia. Misoriented and stray grains can be observed in several parts of sample \#3 (Fig. 14f), which is also caused by the transverse thermal gradient. Because of the high longitudinal thermal gradient, primary dendrites of sample \#5 (Fig. 14h) pack closely and remain underdeveloped.

Figure 15 shows the changes of dendrite spaces from different locations of the blade. The dendrites far from the graphite chill plate show the greater separation distance and coarser structure no matter how thick the wall of the blade. This is because the ceramic core is thinner where the blade wall is thicker and it transmits heat together with the metal material. Also, the ceramic shell, liquid metal and the ceramic core are treated as an ensemble while being cooled by the liquid tin. The size of the ensemble becomes bigger and thicker toward the rabbet of the blade. As the chilling effect and the thermal gradient are reduced, the dendritic structures become coarser gradually.

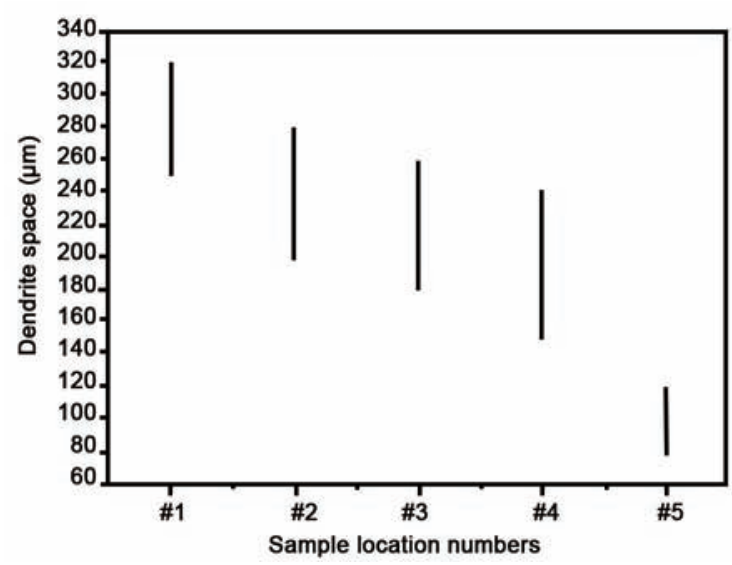

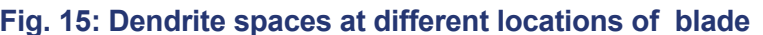

\section{Conclusions}

According to the studies of LMC directional solidification of large-size blades of heavy-duty gas turbine (Grade II), the following conclusions can be obtained:

(1) The hollow directional solidified blade cannot be fully formed when the strength of ceramic shell is too high or too low. Proper strengths of ceramic shell for a well-formed blade can be defined as: $8 \mathrm{MPa}$ of the flexural strength at high temperature $\left(1,550{ }^{\circ} \mathrm{C}\right), 12 \mathrm{MPa}$ of the thermal shock resistance strength and $20 \mathrm{MPa}$ of the residual flexural strength.

(2) The heating temperatures of the mold heating furnace should not be too high or too low. Proper heat preservation temperatures for a well-formed blade are: $1,520^{\circ} \mathrm{C}$ for the upper region and $1,500{ }^{\circ} \mathrm{C}$ for the lower region.

(3) Proper holding time range is 3-5 min, which allows columnar crystal grains to be fine and straight. Too short a standing time can result in a narrow grain of the columnar crystal and a significant deviation of its direction from that of the $<001>$. While, if the holding time is too long (such as 8 min), the grain width of the columnar crystal will be too large and many misoriented grains will form.

(4) When the withdrawal rate (such as 7 and $10 \mathrm{~mm} \cdot \mathrm{min}^{-1}$ ) is greater than the growth rate of the crystal grain, flow wrinkles with different depth occur on the surface of the blade. Proper withdrawal rate (such as 3 and $5 \mathrm{~mm} \cdot \mathrm{min}^{-1}$ ) should be no greater than the growth rate of the crystal grain, to ensure the smooth surface.

(5) The space between dendrites decreases as the blade solidification rate rising, but increases as the distance between the solidification position and the graphite chill plate enlarging. Also, the larger space between dendrites is always accompanied with the coarser dendritic structure, no matter how thick the wall of blade is.

\section{References}

[1] Yutaka K, Toshiharu K, Zhang J X, etc. Development of a nextgeneration Ni-base single crystal superalloy. In: Proceedings of the International Gas Turbine Congress 2003, Tokyo, 2-7 November, 2003: 1-6.

[2] Lebedev A S and Kostennikov S V.Trends in Increasing GasTurbine Units Efficiency.Thermal Engineering, 2008, 55(6): 461468.

[3] Giamei A F, Tschinkel J G. Liquid metal cooling: a new solidification technique. Metall. Trans., 1976, (7A): 1427-1434.

[4] John F, Maxim K. Method and apparatus for casting directionally solidified article. United States Patent: 6311760, 2001.6

[5] Ning Xinglong. New directional solidification technology of single crystal blade. Rare Metal Materials and Engineering, 2001, 30(2): 126.

[6] Jr F X G M, Huang S C, Petterson R J, etc. Liquid metal cooled directional solidification process. United States Patent: 6276433B1, 2001.

[7] Lou Yanchun. Preparation Technology of Ceramic Shell and Core for Directionally Solidified Gas Turbine Blade in Liquid Metal Cooling Process. High-end Formation Manufacturing Technology. Engineering Frontiers Symposium on Advanced Material Processing Technology and through Process Modeling \& Simulation, Beijing, 2012.9.

[8] Gerhard M, Martin V, Gregor H. Large Gas Turbines - the Insurance Aspects: 1-22. 